\title{
Prevalence of Hypoglycemia Among Type 2 Diabetics Undergoing Hemodialysis in a University Hospital
}

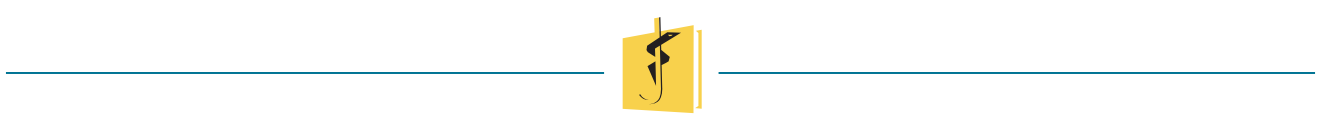

\author{
Maria Concepcion H. Gungon, MD, \\ Elaine C. Cunanan, MD
}

\section{ABSTRACT}

Background: The overall increase in the number of people with diabetes mellitus has a major impact on the increasing number of patients with diabetic kidney disease (DKD). Hypoglycemia that is brought about by altered glucose homeostasis and the hemodialysis process in patients with DKD poses a great challenge to clinicians in achieving blood glucose control. This study has been undertaken to evaluate the prevalence of hypoglycemia among diabetics undergoing hemodialysis and determine the clinical factors predicting the development of hypoglycemia.

Methods: We conducted a prospective, cross-sectional study that included 75 patients with diabetes mellitus undergoing maintenance hemodialysis at the University of Santo Tomas Hospital, Center for Kidney Disease. The patients' predialysis and hourly intradialytic serum glucose levels were measured until the end of the session. Hypoglycemia was considered as a blood glucose $\leq 70 \mathrm{mg} / \mathrm{dL}$ with/without symptoms.

Results: Elderly patients and in-hospital patients tend to develop hypoglycemia with a $p$-value of 0.0028 and 0.001 , respectively. Old age ( $p$-value $0.0093)$, female sex ( $p$-value 0.0224$)$, hypertension (p-value 0.0301), CAD (p-value 0.0058), frequency

Maria Concepcion H. Gungon, MD, FPCP

imapplegungon@yahoo.com.ph

Section of Endocrinology, Diabetes and Metabolism, Department of Internal Medicine, University of Santo

Tomas Hospital, Manila, Philippines of hemodialysis ( $p$-value 0.0000), no caloric intake during the dialysis session ( $p$-value 0.0022) and admission ( $p$-value 0.0007 ) predicted hypoglycemia in our study.

Conclusions: In conclusion, the prevalence of hypoglycemia among patients with type 2 diabetes mellitus undergoing maintenance hemodialysis in our setting is $12 \%$. There is an increased rate of hypoglycemia amongst the elderly as well as admitted patients. Our results identified old age, presence of $C A D$, increased frequency of hemodialysis and hospital admission as the predictors of hypoglycemia.

Keywords: diabetes, hemodialysis, hypoglycemia, $C K D$, end-stage renal disease

\section{INTRODUCTION}

The increasing prevalence of diabetes mellitus has become a global burden and is considered to be a major public health concern. [1] This overall increase in the number of people with diabetes mellitus has influenced the development of diabetic kidney disease (DKD). The leading cause of end-stage renal disease (ESRD) is diabetes, which accounts for $50 \%$ of the cases in the developed world.[2] Chronic kidney disease (CKD) in itself is said to be an independent risk factor for hypoglycemia.[3] Hypoglycemia in patients with DKD could be explained by decreased clearance of hypoglycemic agents and decreased gluconeogenesis by the kidney.[4] In healthy 
individuals, both the liver via glucagon and kidney via catecholamines contribute to the release of glucose into circulation to counterregulate hypoglycemia. Patients with advanced DKD on dialysis behave differently when compared to diabetic patients without kidney disease. More than $40 \%$ of patients with advanced DKD have severe hypoglycemia.[5] It is quite common with the said population since moderate to severe CKD has reduced renal mass, and thus, a reduced capacity for renal glucose release. Malnourishment and muscle wasting present in this population leads to low hepatic glycogen stores and reduces the availability of gluconeogenic substrates. Acidosis, likewise limits the ability of the liver to compensate via hepatorenal reciprocity leading to the development of hypoglycemia.[6]. These factors may contribute to the increased risk of hypoglycemia among patients with CKD and may be an inadvertent consequence of diabetic therapy.

At present, there are no evidence-based guidelines or recommendations in the Philippines about which type of insulin or oral hypoglycemic agents $(\mathrm{OHA})$ to use or avoid depending on the severity of $C K D$. Frequent glucose monitoring may be used to adjust the dosing of insulin and medications to prevent hypoglycemia and caution must be exercised when administering therapy to patients with kidney disease. This study has been undertaken to evaluate the prevalence of hypoglycemia among diabetics undergoing hemodialysis and determine the clinical factors predicting the development of hypoglycemia.

\section{METHODS}

\section{Study Design and Population}

This study was carried out in accordance with the Declaration of Helsinki. The University of Santo Tomas Hospital (USTH) Institutional Review Board (IRB-2016-06-106) approved our protocol. Informed consent was taken from each subject prior to study participation. We conducted a prospective, cross-sectional study which included diabetic patients undergoing maintenance hemodialysis at the USTH Center for Kidney Disease Unit between October 2016 and December 2016. Maintenance dialysis was defined as having received dialysis for more than 60 days. The total number of patients in the hemodialysis unit was 122 and approximately two-third of the patients $(75 / 122)$ had diabetes mellitus. The patients excluded from the study are as follows: patients suffering from starvation (those with severe deficiency in caloric energy intake needed to maintain life), decompensated liver disease (may take the form of any of esophageal variceal bleed, ascites, spontaneous bacterial peritonitis, hepatic encephalopathy, hepatorenal syndrome and hepatocellular carcinoma disease), sepsis and decompensated congestive heart failure (characterized by the development of dyspnea associated with rapid accumulation of fluid within the lung's interstitial and alveolar spaces). The computed sample size was 73 , based on sample size calculation for a multiple regression study (given the desired probability level of 0.05 , anticipated effect size of 0.20 and desired statistical power level of 0.15). We were able to extract data regarding demographic variables and drug prescriptions for the period between October 2016 and December 2016. The patients did not change their dietary habits or drug treatment before or during the days of the study.

Predialysis and hourly intradialytic capillary blood glucose (CBG) levels were measured using a calibrated glucose meter, Freestyle Optium Neo. The Free Style Optium Neo system met the requirements for accuracy in user performance testing and trained operator system accuracy evaluation of ISO 15197:2013. The device can measure results ranging from 20-500 mg/dL. Hypoglycemia was considered based on the definition given by the American Diabetes Association. [7]

Hypoglycemia alert value (level 1): $\leq 70 \mathrm{mg} /$ $\mathrm{dL}(3.9 \mathrm{mmol} / \mathrm{L})$, sufficiently low for treatment with fast-acting carbohydrate and dose adjustment of glucose-lowering therapy

Clinically significant hypoglycemia (level 2): $\leq 54 \mathrm{mg} / \mathrm{dL}(3.0 \mathrm{mmol} / \mathrm{L})$, sufficiently low to indicate serious, clinically important hypoglycemia

Severe hypoglycemia (level 3): No specific glucose threshold; hypoglycemia associated with severe cognitive impairment requiring external assistance for recovery.

Neuroglycopenic manifestations of hypoglycemia include behavioral changes, confusion, psychomotor abnormalities and at lower levels, seizure and coma. Neurogenic symptoms include palpitations, tremor, anxiety, sweating, hunger and paresthesias. Signs of hypoglycemia include pallor, diaphoresis, tachycardia and raised systolic blood pressure. 
[5] The researchers followed the patients closely to identify any signs or symptoms of hypoglycemia during the dialysis session. If the patient developed symptoms and signs of hypoglycemia and/or CBG reached $\leq 70 \mathrm{mg} / \mathrm{dL}$, additional CBG monitoring for every 15 minutes was facilitated until normalization of glucose was achieved. Patients with hypoglycemia were medicated with $50 \mathrm{~mL}$ of $50 \%$ glucose solution intravenously.

\section{Statistical Analysis}

All data were processed using the SPSS Statistical Package version 23.02015 (SPSS Inc., Chicago, IL). Data were presented as frequency (percentage) and mean and standard deviation at baseline characteristics. Chi-square test and T-test were computed to compare the demographics and medical profiles of those patients with and without hypoglycemia. The predictors of hypoglycemia were identified based on calculation of regression analysis. A significant decrease in mean $C B G$ in different time periods was calculated using Repeated Measures Analysis of Variance (RM-ANOVA) with post-hoc Scheffe's test to determine significant groups. A p-value of $<0.05$ was considered statistically significant in this study.

\section{RESULTS}

A total of 75 patients were included in the study, 9 of whom developed hypoglycemia during the data collection. All the patients who had hypoglycemic values were asymptomatic. The results of patients' demographic and clinical parameters were listed in Table 1. The participants' age ranged from 41 to 86 years old, a majority of which were elderly, with a computed mean age of 69.50 years old. More than half $(54.67 \%)$ of the participants were females. Baseline comorbidities were hypertension and coronary artery disease comprising of $89 \%$ and $32 \%$ of the participants, respectively. A majority of the patients $(52 \%)$ were not on any oral hypoglycemic agent or insulin. Of the 75 patients, 22 patients $(29.3 \%)$ were on monotherapy with dipeptidyl-peptidase-4 inhibitors (DPP4 inhibitors). Most of the patients included in the study were diagnosed with diabetes for more than 10 years (12.93 \pm 9.31) and were already on maintenance hemodialysis for more than 2 years $(2.51 \pm 2.49)$. Based
Table 1. Baseline characteristic and demographics of the diabetic patients undergoing maintenance hemodialysis at USTH-CKD

\begin{tabular}{|c|c|}
\hline Variables & $\begin{array}{l}\text { Subjects } \\
\mathrm{n}=75\end{array}$ \\
\hline Age (years, $X \pm S D)^{a}$ & $69.50 \pm 9.73$ \\
\hline \multicolumn{2}{|l|}{ Gender $(\mathrm{F} \%)^{b}$} \\
\hline Female & $41(54.67)$ \\
\hline Male & $34(45.33)$ \\
\hline $\mathrm{BMI}\left(\mathrm{kg} / \mathrm{m}^{2)}(\mathrm{X} \pm \mathrm{SD})^{\mathrm{a}}\right.$ & $22.75 \pm 10.53$ \\
\hline \multicolumn{2}{|l|}{ Hypertension (F \%) } \\
\hline Positive & $67(89.33)$ \\
\hline Negative & $8(10.67)$ \\
\hline \multicolumn{2}{|l|}{ CAD (F \%) ${ }^{b}$} \\
\hline Positive & $24(32.00)$ \\
\hline Negative & $51(68.00)$ \\
\hline Duration of CKD (years, $X \pm S D$ ) a & $2.51 \pm 2.49$ \\
\hline Duration of diabetes (years, $X \pm S D)^{a}$ & $12.93 \pm 9.31$ \\
\hline \multicolumn{2}{|l|}{ DM medications $(\mathrm{F} \%)^{\mathrm{b}}$} \\
\hline DPP-4 inhibitors & $22(29.33)$ \\
\hline Insulin & $6(8.00)$ \\
\hline Both & 8 (10.67) \\
\hline None & $39(52.00)$ \\
\hline $\begin{array}{l}\text { Frequency of hemodialysis } \\
\qquad(H D / \text { week, } X \pm S D)^{a}\end{array}$ & $2.75 \pm 0.54$ \\
\hline \multicolumn{2}{|l|}{ Blood pressure $(\mathrm{mmHg}, \mathrm{X} \pm \mathrm{SD})^{\mathrm{a}}$} \\
\hline Systole & $135 \pm 10.71$ \\
\hline Diastole & $82.83 \pm 8.32$ \\
\hline \multicolumn{2}{|l|}{$\operatorname{Diet}(F \%)^{b}$} \\
\hline Ate & $75(100)$ \\
\hline Did not eat & 0 \\
\hline \multicolumn{2}{|l|}{ Admission $(F, \%)^{b}$} \\
\hline Admitted & $5(6.67)$ \\
\hline Outpatient & $70(93.33)$ \\
\hline
\end{tabular}

a. $X+S D$ values means mean $(X)$ and standard deviation (SD) b. $F$ means frequency, $\%$ means percentage.

on the patients' computed body mass index (AsiaPacific task force obese classification), 9.33\% were underweight, $49.33 \%$ were normal, $17.33 \%$ were overweight and $24 \%$ were categorized as obese.

A comparison of the general characteristics, clinical parameters and hypoglycemic medications between hypoglycemic and non-hypoglycemic patients were shown in Table 2. Patients were divided into two groups, those with hypoglycemia and without 
Table 2. General characteristics, clinical parameters and hypoglycemic medications of diabetic patients undergoing maintenance hemodialysis at USTH-CKD with and without hypoglycemia

\begin{tabular}{|c|c|c|c|}
\hline \multirow{2}{*}{ Variables } & With Hypoglycemia ${ }^{a}$ & Without Hypoglycemia ${ }^{a}$ & \multirow{2}{*}{ P-value } \\
\hline & $9(12 \%)$ & $66(88 \%)$ & \\
\hline Age (years) & $75.0 \pm 7.92$ & $64.0 \pm 11.53$ & 0.0028 \\
\hline \multicolumn{4}{|c|}{ Gender } \\
\hline Female & $6(66.67)$ & $35(53.03)$ & 0.441 \\
\hline Male & $3(33.33)$ & $31(46.97)$ & \\
\hline BMI $\left(\mathrm{kg} / \mathrm{m}^{2}\right)$ & $22.24 \pm 6.27$ & $23.26 \pm 4.26$ & 0.6422 \\
\hline \multicolumn{4}{|c|}{ Hypertension } \\
\hline Positive & $8(88.89)$ & 59 (89.39) & 0.963 \\
\hline Negative & $1(11.11)$ & $7(10.61)$ & \\
\hline \multicolumn{4}{|c|}{ CAD } \\
\hline Positive & $3(33.33)$ & $21(31.82)$ & 0.927 \\
\hline Negative & $6(66.67)$ & $45(68.18)$ & \\
\hline Duration of CKD (years) & $2.33 \pm 2.56$ & $2.68 \pm 2.41$ & 0.7017 \\
\hline Duration of diabetes (years) & $14.25 \pm 9.99$ & $11.60 \pm 8.63$ & 0.4656 \\
\hline \multicolumn{4}{|c|}{ DM Medications } \\
\hline DPP4 inhibitors & $2(22.22)$ & $20(30.30)$ & 0.955 \\
\hline Insulin & $1(11.11)$ & $5(7.58)$ & \\
\hline Both & $1(11.11)$ & $7(10.61)$ & \\
\hline None & $5(55.56)$ & $34(51.52)$ & \\
\hline Frequency of hemodialysis (per week) & $2.67 \pm 0.50$ & $2.82 \pm 0.58$ & 0.4211 \\
\hline \multicolumn{4}{|c|}{ Blood Pressure $(\mathrm{mmHg})$} \\
\hline Systole & $133.33 \pm 10.00$ & $136.67 \pm 11.41$ & 0.3766 \\
\hline Diastole & $84.44 \pm 7.26$ & $81.21 \pm 9.37$ & 0.2513 \\
\hline \multicolumn{4}{|c|}{ Diet } \\
\hline Ate & $9(100)$ & $66(100)$ & 1 \\
\hline Did not eat & $0(0)$ & $0(0)$ & \\
\hline \multicolumn{4}{|c|}{ Admission } \\
\hline Inpatient & $3(33.33)$ & $3(33.33)$ & \multirow{2}{*}{0.001} \\
\hline Outpatient & $6(66.67)$ & $6(66.67)$ & \\
\hline
\end{tabular}

a. Data are expressed as mean \pm standard deviation

b. Significant difference if $P$ value is $<0.05$

hypoglycemia. Older patients tend to develop and experience hypoglycemia with a p-value of 0.0028 . Hospitalized patients were found to have a higher tendency to develop hypoglycemia, reaching statistical significance with a p-value of 0.001 . Three patients $(4 \%)$ taking oral hypoglycemic agents and/or injecting insulin developed hypoglycemia during the observational period. Five patients $(6.67 \%)$ developed hypoglycemia even without $\mathrm{OHA}$ /insulin injection. The use of insulin as well as OHA did not reach statistical difference to cause hypoglycemia compared to patients with no maintenance medication.
Predictors of hypoglycemia were identified based on calculation of regression analysis as demonstrated in Table 3. Older age ( $p$-value 0.0093), female sex (p-value 0.0224), hypertension (p-value 0.0301), CAD (p-value 0.0058), frequency of hemodialysis ( $p$-value 0.0000), no food intake during the dialysis session (p-value 0.0022) and admission ( $p$-value 0.0007 ) predicted hypoglycemia in our study.

As noted in Table 4, there was an observation of downward trend in the mean predialysis CBG in comparison to the mean intradialysis $C B G$ with a p-value of 0.0000 . 
Table 3. Predictors of hypoglycemia among diabetics undergoing maintenance hemodialysis at USTH-CKD

\begin{tabular}{lcccc}
\hline Variables & Coefficients & Standard Error & t-Stat & P-value $^{\alpha}$ \\
\hline Age & -0.0087 & 0.0032 & -2.6767 & 0.0093 \\
Sex & 0.1768 & 0.0757 & 2.3360 & 0.0224 \\
BMI kg/m & 0.0267 & 0.0157 & 1.7049 & 0.0927 \\
Hypertension & 0.3123 & 0.1409 & 2.2167 & 0.0301 \\
CAD & 0.2724 & 0.0955 & 2.8531 & 0.0058 \\
Duration of CKD & 0.0076 & 0.0222 & 0.3413 & 0.7340 \\
Duration of Diabetes & -0.0007 & 0.0054 & -0.1219 & 0.9033 \\
Frequency of HD & 0.3454 & 0.0602 & 5.7380 & 0.0000 \\
Diet & 0.8909 & 0.2804 & 3.1775 & 0.0022 \\
Admitted/Outpatient & 0.5091 & 0.1440 & 3.5342 & 0.0007 \\
\hline
\end{tabular}

a. Significant difference if $P$ value is $<0.05$

Table 4. Mean $C B G$ and percentage drop of $C B G$ in different time period among diabetic patients undergoing maintenance hemodialysis at USTH-CKD

\begin{tabular}{|c|c|c|c|c|c|c|c|}
\hline & & Pre-HD & lst Hour & 2nd Hour & 3rd Hour & 4th Hour & P-value ${ }^{b}$ \\
\hline \multirow[t]{2}{*}{ Overall } & $\begin{array}{l}\text { Mean } \\
+\mathrm{SD}^{a}\end{array}$ & $\begin{array}{r}151.28 \\
+81.76\end{array}$ & $\begin{array}{r}131.95 \\
+59.71\end{array}$ & $\begin{array}{r}117.89 \\
+33.46\end{array}$ & $\begin{array}{r}117.89 \\
+33.46\end{array}$ & $\begin{array}{r}112.04 \\
+25.05\end{array}$ & 0.0000 \\
\hline & $\%$ decrease & \multicolumn{2}{|c|}{$15.00 \%$} & $13.00 \%$ & $-1.00 \%$ & $5.00 \%$ & \\
\hline
\end{tabular}

a. Data are expressed as mean \pm standard deviation

b. Significant difference if $P$ value is $<0.05$

\section{DISCUSSION}

The study shows that in our clinical setting, up to $12 \%$ of patients with type 2 diabetes mellitus developed hypoglycemia during hemodialysis. One retrospective cohort study revealed a higher incidence of hypoglycemia seen in patients with CKD versus without CKD. Among patients with diabetes, the rate was 10.72 versus 5.33 per 100 patient-months for CKD versus no CKD, respectively. [3] A higher prevalence rate of $52.9 \%$ was observed in another study done by Chiao-Yin Sun, et al.[8] The study participants were stable diabetic hemodialysis patients taking pharmacological therapy. Hypoglycemia was likewise set at $\leq 70 \mathrm{mg} / \mathrm{dL}$ for those two studies. CKD is a significant risk factor for the development of hypoglycemia with or without the presence of diabetes, but the risk is greatest in patients with CKD and diabetes. [3] Conditions that are associated with an increased risk of hypoglycemia in these patients include decreased gluconeogenesis in the remnant kidneys, deranged metabolic pathways, inadequate nutrition, decreased insulin clearance, glucose loss to the dialysate and diffusion of glucose into erythrocytes during hemodialysis. [9]
It is also interesting to mention that a majority of the study participants (52\%) were not on oral hypoglycemic agents or insulin injections. Recently, it has been reported that in up to one-third of diabetic dialysis patients with the presumptive diagnosis of diabetic nephropathy, glycemic control improves spontaneously with progression of CKD, loss of residual renal function and initiation of dialysis therapy. This led to normalization of hemoglobin A $1 \mathrm{c}(<6 \%)$ and glucose levels causing discontinuation of insulin or other diabetic medications.[10] Whether the 52\% of participants belonged to this so-called "burnt-out diabetes" group needs to be further studied.

Another finding in our study revealed that neither OHA nor insulin posed a greater risk of causing hypoglycemia. Out of the 36 patients who were on either insulin or oral therapy, only 4 patients developed hypoglycemia and surprisingly a higher number of patients (5 patients) developed hypoglycemia even without medication. This is likewise evident in the previous study by Chiao-Yin Sun, et al., wherein they found out that OHA, specifically meglitinide and glipizide are as safe as insulin in diabetic hemodialysis patients. [8] The most favored antidiabetic agent in our setting was a DPP-4 inhibitor. 
Twenty-two percent of the participants were on monotherapy with DPP-4 inhibitor and $10.7 \%$ were on combination therapy of DPP-4 inhibitor with basal insulin. In a meta-analysis done by Nakamura, et al., wherein they evaluated the therapeutic effects of DPP-4 inhibitors in hemodialysis patients, no patient showed severe side effects such as hypoglycemia and liver dysfunction. They concluded that these adverse events resulting from DPP-4 inhibitor treatments do not occur at a higher incidence than in hemodialysis patients. [1 1]

Another finding in our study revealed that admitted patients tend to develop hypoglycemia with a p-value of 0.001 . This is concurrent with the study done by Kagansky, et al.[12] Hospitalized patients 70 years or older showed that hypoglycemia is a common complication in this age group with an incidence of $5.2 \%$. Another study done by Finfer, et al.,[13] reported a higher prevalence of hypoglycemia, in this subset of patients, since they were more sick and on more aggressive glycemic regimen. It goes to show that the prevalence and incidence of hypoglycemia varies depending upon the in-patient setting in question and the glycemic threshold used in each study. Admitted patients were at a higher risk for hypoglycemia due to the following reasons: intensive glucose management, more stringent capillary glucose monitoring, presence of hypoglycemia associated autonomic failure, organ failure and polypharmacy.[14] A reduced caloric intake may also play a role in patients who are ill since most of them suffer with anorexia, nausea and vomiting, making them more prone to develop hypoglycemia.

Predictors of hypoglycemia identified in our study based on the calculation of regression analysis include old age (p-value 0.0093), female sex (p-value $0.0224)$, comorbidities like hypertension ( $p$-value $0.0301), C A D$ (p-value 0.0058), increased frequency of hemodialysis ( $p$-value 0.0000), no food intake during the dialysis session (p-value 0.0022) and admission (p-value 0.0007). Age appears to affect counterregulatory responses to hypoglycemia aside from the presence of several predisposing factors such as comorbidity, polypharmacy, chronic renal and hepatic impairment and poor nutrition. Advanced age has been a contributing factor to hypoglycemia as also depicted in the ACCORD and ADVANCE trials. Female sex likewise predicted hypoglycemia in our study. Davis, et al.,[15] also reported the same finding of hypoglycemia in women.
It may be related to sex differences in counterregulatory responses to hypoglycemia seen in the female sex. Frequency of hemodialysis predicted the development of hypoglycemia during the conduct of our study. The more frequent the dialysis session of the patient, the higher rate of hypoglycemia noted. The nutritional status of the patients were assessed by computing the body mass index, which did not show statistical significance in predicting hypoglycemia, however, our study revealed that no caloric intake during hemodialysis predicted hypoglycemia. It was studied by Kalantar-Zadeh, et Al.,[16] that intradialytic meals or oral nutritional supplements to dial$y$ sis patients and other nutritional interventions are the most promising intervention to increase serum albumin and improve longevity and quality of life in this patient population. However, nephrologists from the USA refused intradialytic meals due postprandial hypotension, risk of choking or aspiration, infection control and hygiene issues, including fear of fecal-oral transmission of diseases such as hepatitis $A$, staff burden and distraction, and diabetes and phosphorus control. A larger study might be done to assess the applicability of intradialytic meals in preventing hypoglycemia in this population.

During the conduct of the study, we observed that there is a decrease in mean CBG from pre-hemodialysis to the intradialytic CBG with a p-value of 0.0000 . The decreasing trend was likewise observed in a study done by Burmeister, et al., indicating a higher risk of intradialytic hypoglycemia for diabetic individuals who presumably present the best glycemic control, especially when in glucose-free dialysis. Further, they found out a significant decrement of blood glucose levels during dialysis which they believed to be due to loss of glucose in the dialysate.[17]

The presence of hypoglycemia unawareness in our study population was also observed with no participants manifesting neuroglycopenic or neurogenic symptoms despite having CBG values $\leq 54 \mathrm{mg} / \mathrm{dL}$. In a study done by Gerich, et al., it was reported to be found in $25 \%$ of patients with diabetes. [18]

There are some inherent limitations to consider in our study. The total study population is small and the duration of the study is limited to three months. A larger sample size and a longer timeframe (for example, one year) would have identified the true prevalence of hypoglycemia in our setting. We also used glucose meter as a tool to determine hypogly- 
cemia. A variety of factors can affect glucose meter results, including operator technique, environmental exposure, and patient factors, such as medication, oxygen therapy, anemia, hypotension and other disease states. Ideally, the use of plasma to confirm hypoglycemia should have been facilitated.

\section{CONCLUSIONS}

The prevalence of hypoglycemia in diabetic patients undergoing maintenance hemodialysis at USTH CKD is $12 \%$. Admitted patients and elderly individuals reported association in the development of hypoglycemia; hence, more frequent point-of-care testing is advised to these patients during their scheduled dialysis sessions. Our study identified old age, presence of CAD, increased frequency of hemodialysis, no caloric food intake and hospital admission as predictors of hypoglycemia.

\section{Conflict of Interest}

No potential conflict of interest relevant to this article was reported.

\section{Acknowledgments}

No funding was required in our study. It is with the support and generosity of the Section of Endocrinology, Diabetes and Metabolism and St. Thomas Diabetes Center of the University of Santo Tomas Hospital that this manuscript was accomplished. We thank our consultants from the Section of Endocrinology, Diabetes and Metabolism of USTH, especially Dr. Maria Honolina Gomez, our Research Committee Head, who provided insight and expertise that greatly assisted the research and improved the manuscript. We also would like to acknowledge consultants and fellows from the Section of Nephrology and Center for Kidney Disease for allowing us to conduct our study on their patients. 


\section{REFERENCES}

1. World Health Organization Western Pacific Region. Philippines: health situation and trend: communicable and noncommunicable diseases, health risk factors and transition. Manila, Philippines. World Health Organization Regional Office for the Western Pacific. Available at: http://www. wpro.who.int/publications/CHIPS2010.pdf; 2010.

2. Tuttle KR, Bakris GL, Bilous RW, Chiang JL, de Boer $I H$, Goldstein-Fuchs J, et al. Diabetic kidney disease: A report from an ADA consensus conference, diabetes care. 2014 Oct;37(10):2864-83. Available at: https://doi. org/10.2337/dc14-1296.

3. Moen MF, Zhan M, Hsu VD, Walker LD, Einhorn LM, Seliger SL, et al. Frequency of hypoglycemia and its significance in chronic kidney disease. Clin J Am Soc Nephrol. 2009;4:1121-7.

4. National Kidney Foundation. KDOQI clinical practice guideline for diabetes and CKD: 2012 update. Am J Kidney Dis. 2012;60:850-86.

5. Rhee CM, Leung AM, Kovesdy CP, Lynch KE, Brent GA, Kalantar-Zadeh $\mathrm{K}$, et al. Updates on the management of diabetes in dialysis patients. Semin Dial. 2014;27:13545. doi: $10.1111 /$ sdi.12198

6. Woerle HJ, Meyer C, Popa EM, Cryer PE, Gerich JE. Renal compensation for impaired hepatic glucose release during hypoglycemia in type 2 diabetes: Further evidence for hepatorenal reciprocity. Diabetes. 2003;52:1386-92.

7. International Hypoglycaemia Study Group. Glucose concentrations of less than $3.0 \mathrm{mmol} / \mathrm{L}(54 \mathrm{mg} / \mathrm{dL})$ should be reported in clinical trials: a joint position statement of the American Diabetes Association and the European Association for the Study of Diabetes. Diabetes Care. 2017;40:155-7.

8. Chiao-Yin S, Chin-Chan L, Wu MS. Hypoglycemia in diabetic patients undergoing chronic hemodialysis. Therapeutic apheresis and dialysis. 2009 Apr; 13(2):95-102.

9. Abe M, Kalantar-Zadeh K. Haemodialysis-induced hypoglycaemia and glycaemic disarrays. Nature Reviews Nephrology. 2015;11:302-13.

10. Kalantar-Zadeh, Derose SF, Nicholas S, Benner D, Kumar Sharma, Kovesdy CP. Burnt-out diabetes: The impact of CKD progression on the natural course of diabetes mellitus. J Ren Nutr. 2009 Jan; 19(1):33-37.
11. Nakamura $Y$, Hasegawa $H$, Tsuji M, Udaka $Y$, Mihara $M$, Shimizu $T$, et al. Diabetes therapies in hemodialysis patients: Dipeptidase-4 inhibitors. World J Diabetes. 2015 Jun $25 ; 6(6): 840$.

12. Kagansky N, Levy S, Rimon E, Cojocaru L, Fridman A, Ozer $\mathrm{Z}$, Knobler H. Hypoglycemia as a predictor of mortality in hospitalized elderly patients. Arch Intern Med. 2003 Aug 1 1-25; 163(15): 1825-9.

13. NICE-SUGAR Study Investigators. Finfer S, Liu B, Chittock DR, Norton R, Myburgh JA, et al. Hypoglycemia and risk of death in critically ill patients. N Engl J Med. 2012;367(12): 1 108-118.

14. Hulkower RD, Pollack RM, Zonsnein J. Understanding hypoglycaemia in hospitalized patients. Diabetes Management (Lon). 2014 Mar;4(2): 165-76.

15. Davis SN, Fowler S, Costa F. Hypoglycemic counterregulatory responses differ between men and women with type 1 diabetes. Diabetes. 2000;4965-72.

16. Kalantar-Zadeh K, Ikizler T. Let them eat during dialysis: An overlooked oppurtunity to improve outcome in maintenance hemodialysis patients. J Ren Nutr. 2013 May;23(3): 157-163.

17. Burmeister JE, Scapini A, da Rosa Miltersteiner D, da Costa MG, Campos BM. Glucose-added dialysis fluid prevents asymptomatic hypoglycaemia in regular haemodialysis. Nephrol Dial Transplant. 2007;22:1184-9.

18. Gerich JE, Mokan M, Veneman T, Korytkowski M, Mitrakou A. Hypoglycemia unawareness. Endocr Rev. 1991 Nov; 12(4):356-71.

(i) Open Access This article is licensed under a By Creative Commons Attribution 4.0 International License, which permits use, sharing, adaptation, distribution and reproduction in any medium or format, as long as you give appropriate credit to the original author(s) and the source, provide a link to the Creative Commons license, and indicate if changes were made. The images or other third party material in this article are included in the article's Creative Commons license, unless indicated otherwise in a credit line to the material. If material is not included in the article's Creative Commons license and your intended use is not permitted by statutory regulation or exceeds the permitted use, you will need to obtain permission directly from the copyright holder. To view a copy of this license, visit http://creativecommons.org/licenses/by/4.0/. 